\title{
Canadian Association of Emergency Physicians sepsis treatment checklist: optimizing sepsis care in Canadian emergency departments
}

\author{
Dennis Djogovic, MD; Robert Green, MD; Robert Keyes, MD; Sara Gray, MD; Robert Stenstrom, MD; \\ David Sweet; Jonathan Davidow, MD; Edward Patterson, MD; David Easton, MD; \\ Shavaun MacDonald, MD; Jonathan Gaudet; Michael R. Kolber, MD; David Lechelt, MD; \\ Daniel Howes, MD, on Behalf of the CAEP Critical Care Practice Committee
}

\section{ABSTRACT}

Objective: The Canadian Association of Emergency Physicians (CAEP) sepsis guidelines created by the CAEP Critical Care Practice Committee (C4) and published in the Canadian Journal of Emergency Medicine (CJEM) form the most definitive publication on Canadian emergency department (ED) sepsis care to date. Our intention was to identify which of the care items in this document are specifically necessary in the ED and then to provide these items in a tiered checklist that can be used by any Canadian ED practitioner.

Methods: Practice points from the CJEM sepsis publication were identified to create a practice point list. Members of $\mathrm{C} 4$ then used a Delphi technique consensus process over May to October 2009 via e-mail to create a tiered checklist of sepsis care items that can or could be completed in a Canadian ED when caring for the septic shock patient. This checklist was then assessed for use by a survey of ED practitioners from varying backgrounds (rural ED, community ED, tertiary ED) from July to October 2010.

Results: Twenty sepsis care items were identified in the CAEP sepsis guidelines. Fifteen items were felt to be necessary for ED care. Two levels of checklists were then created that can be used in a Canadian ED. Most ED physicians in community and tertiary care centres could complete all parts of the level I sepsis checklist. Rural centres often struggle with the ability to obtain lactate values and central venous access. Many items of the level II sepsis checklist could not be completed outside the tertiary care centre ED.

Conclusion: Sepsis care continues to be an integral and major part of the ED domain. Practice points for sepsis care that require specialized monitoring and invasive techniques are often limited to larger tertiary care EDs and, although heavily emphasized by many medical bodies, cannot be reasonably expected in all centres. When the resources of a centre limit patient care, transfer may be required.
Keywords: checklist, emergency department, sepsis

Emergency department (ED) sepsis care is important and reduces mortality. Provision of this care can be improved through the use of sepsis protocols or guidelines. ${ }^{1-4}$ The Canadian Association of Emergency Physicians (CAEP) sepsis guidelines were published in 2008 to that end. ${ }^{5}$ Although meant to be all-encompassing and to present current "state of the art" care, some have requested a more user-friendly format. ${ }^{6}$ As well, controversy exists as to whether specialized invasive sepsis care is necessary in the ED.? ED care in Canada varies, depending on patient location, access to tertiary care, ED volumes, time of operation, and practitioner access to treatment/monitoring skills and capabilities. ${ }^{8}$ At present, no publication has made an attempt to present sepsis care protocols based on the resources available within differing types of EDs.

To meet the needs of all Canadian ED physicians, the CAEP Critical Care Practice Committee (C4) sought to take the CAEP sepsis guidelines it created and compile two checklists of care items. Although all items are felt to be necessary for optimal sepsis management, having two checklists with escalating levels of care should allow physicians in any Canadian ED to use the checklist that is more appropriate for their abilities and resources. ED sepsis care should be of short duration ( $<6$ hours) and attempt to ensure timely transfer to the most appropriate location for ongoing care (e.g., operating room, tertiary hospital,

Correspondence to: Dr. Dennis Djogovic, Office 1G1.59, Walter MacKenzie Health Sciences Centre, 8440-112 Street, Edmonton AB, T6G 2B7; djogovic@ualberta.ca.

This article has been peer reviewed. 
intensive care unit [ICU]). Remaining items were felt to be more important for inpatient care.

\section{METHODS}

The membership of $\mathrm{C} 4$ consists of 11 physicians who practice emergency medicine in hospitals that vary in size, location, and level of specialized care and who have a major interest or training in critical care medicine. The $\mathrm{C} 4$ chair created the checklists, compiled the results, and reported results and comments from each round to the collective group but did not participate in voting or determination of checklist items.

C4 enacted the Delphi technique, a widely used and accepted method of gathering data from participants within their domain of expertise, to bring structure and credibility to consensus-building efforts. Created in the 1950s, the Delphi technique has been used in fields such as program planning, needs assessment, policy determination, and resource use. The aim was to achieve a consensus of opinion on a specific issue via multiple iterations of questionnaires. ${ }^{9-11}$ As $\mathrm{C} 4 \mathrm{mem}-$ bers live across Canada, it was felt that the Delphi technique would best draw in the collective opinions and experience of this diverse group.

Twenty items were identified in the CAEP C4 sepsis document that address potential management options in severe sepsis. As a first step, C4 identified which items should be part of the ED focus of care of the septic patient. Consensus was determined by $\geq 80 \%$ agreement. To be identified as an ED there needed to be 24-hour in-house physician coverage where sepsis care would be $<6$ hours in duration.

Further Delphi consensus process was conducted to identify which items were 1 ) reasonable to complete in any Canadian ED (level I) and 2) more challenging to achieve in the ED but should be achieved if at all possible (level II).

Once the two escalating levels of sepsis care checklists were created, input was sought from non-C4 ED physicians of varying backgrounds. A survey containing a brief case scenario describing a septic shock patient, followed by the two checklists of care items, was distributed. ED physicians were asked to identify which care items they could and could not complete in their institution, identified as a rural ED, a community ED, or a tertiary ED. Anonymous results were then collated by the $\mathrm{C} 4$ chair. Definitions for rural, community, and tertiary EDs were not strictly defined or enforced but were assumed to be as follows: rural EDs were departments outside city limits that do not have an ICU, and community and tertiary EDs were differentiated based on ability to have a full range of supportive services (e.g., ICU, dialysis, cardiac catheterization).

\section{RESULTS}

The first round of the Delphi consensus process identified 15 of 20 sepsis care items in the document published in the Canadian Fournal of Emergency Medicine $e^{5}$ that should be managed within an ED. One of the five items, glucose control, was initially voted to be an ED care item. However, comments submitted from voting members did indicate a consensus about this conclusion. A further round of questioning addressing this specific issue resulted in this item secondarily reaching consensus as an inpatient care item.

A second round of consensus process demonstrated that of the $15 \mathrm{ED}$ focus of care items, 8 care items attained consensus for the level I checklist (reasonable to achieve items), whereas 5 items attained consensus to form the level II checklist (attempt to achieve if possible items). Two items that did not receive consensus for either checklist were defaulted to the level II checklist, bringing the number of care items in that checklist to seven (Table 1).

Once the Delphi consensus process was complete, a tiered treatment checklist was compiled that contained 1) a definition of identification of the septic patient, 2) a level I checklist of the most reasonable to achieve items in the ED, and 3) a level II checklist of more challenging but necessary items to try to achieve in the ED (Table 2).

With the checklists complete, a survey containing a descriptive patient case scenario and a list of the sepsis care items was distributed to ED physicians. Physicians were asked to identify which items of care they could provide in their ED (rural, community, or tertiary). A total of 124 surveys were returned from 20 rural ED physicians, 23 from community ED physicians, and 81 from tertiary ED physicians. All community and tertiary ED physicians could complete all items of the level I checklist. Almost 100\% of rural ED physicians could complete all parts of the basic checklist except for obtaining lactate values ( 6 of 20 , 


\section{Table 1. Delphi consensus results}

Round 1 results: sepsis care items felt to be inpatient care items Arterial line placement

Provision of recombinant activated protein $\mathrm{C}$

Positive pressure ventilation

Low tidal volume ventilation

Intensive glucose control

Round 2 results: sepsis care items felt to be ED care items

A) Level I checklist: "reasonable to achieve" care items

Endotracheal intubation if needed

Central venous line access if needed

Early aggressive fluid resuscitation

Vasopressor administration to achieve MAP > $65 \mathrm{~mm} \mathrm{Hg}$ and end-organ perfusion

Serum lactate values

Antimicrobial administration within 60 minutes of sepsis recognition

Antimicrobials based on likely pathogens and patient and local epidemiology

Microbial cultures as soon as septic patient identified but not delaying treatment

B) Level II checklist: "try to achieve if possible" care items

Central venous pressure (CVP) monitoring

Central venous gas oxygen saturation $\left(\mathrm{CVO}_{2}\right.$ sat $)$

Fluid resuscitation guided by CVP 8-12 $\mathrm{mm} \mathrm{Hg}$

Inotrope administration to achieve $\mathrm{CVO}_{2}$ sat $>70 \%$ if adequate cardiac output (MAP > $65 \mathrm{~mm} \mathrm{Hg}$, CVP 8-12 mm Hg, Hct > $30 \%$, and adequate urine output)

Blood transfusion for CVP 8-12 $\mathrm{mm} \mathrm{Hg}$ and $\mathrm{CVO}_{2}$ sat $<70 \%$ to target Hct $>30 \%$

Consensus not reached so defaulted to level II care items Attempt infectious source control

Steroid administration in patients not responsive to volume and/ or vasopressor use

$\mathrm{ED}=$ emergency department; $\mathrm{Hct}=$ hematocrit; $\mathrm{MAP}=$ mean arterial pressure

$30 \%)$ and central venous line access (11 of 20, 55\%). Most items of the level II checklist could not be obtained by either rural or community ED physicians. All items of the level II checklist could be obtained by $>80 \%$ of tertiary ED physicians, but even these results were not universally obtainable in the tertiary care ED (Table 3).

\section{DISCUSSION}

Sepsis care continues to be at the forefront of the critical care and emergency medicine literature. The CAEP Critical Care Practice Committee is committed to providing not only state of the art information for CAEP members but also utility and guidance in how to use this valuable information. Like Canada itself, C4 members are diverse and spread across a vast area. Using

\section{Table 2. CAEP sepsis treatment checklist}

Suspect severe sepsis or septic shock in a patient who has

1. Suspected or confirmed source of infection

2. Any of the following:

a. SBP $<90 \mathrm{~mm} \mathrm{Hg}$ after $1-2 \mathrm{~L}$ of rapidly administered crystalloid

b. Lactate $>4 \mathrm{mmol} / \mathrm{L}$

c. Significant new-onset organ dysfunction

And institute a CAEP ED sepsis treatment checklist:

Level I checklist: reasonable to Level II checklist: try to achieve if achieve items

Endotracheal intubation if

needed possible items

Central venous line access if needed*

Central venous pressure monitoring

Early aggressive fluid resuscitation

Vasopressor administration to achieve MAP > $65 \mathrm{~mm}$

$\mathrm{Hg}$ and end-organ perfusion

Central venous gas oxygen saturation

Fluid resuscitation guided by CVP 8-12 mm Hg

Inotrope administration to achieve $\mathrm{CVO}_{2}$ sat $>70 \%$ if adequate cardiac output (MAP $>65 \mathrm{~mm} \mathrm{Hg}$, CVP 8-12 mm $\mathrm{Hg}$, Hct $>30 \%$, and adequate urine output)

Serum lactate values* Blood transfusion for CVP 8$12 \mathrm{~mm} \mathrm{Hg}$ and $\mathrm{CVO}_{2}$ sat < $70 \%$ to target Hct $>30 \%$

Antimicrobial administration within 60 minutes of sepsis recognition

Antimicrobials based on likely pathogens and patient and local epidemiology

Attempt infectious source control

Steroid administration in patients not responsive to volume and/or vasopressor use

Microbial cultures as soon as septic patient identified but not delaying treatment

$\mathrm{CAEP}=$ Canadian Association of Emergency Physicians; $\mathrm{CVO}_{2}$ sat $=$ central venous gas oxygen saturation; $\mathrm{CVP}=$ central venous pressure; $\mathrm{ED}=$ emergency department; $\mathrm{Hct}=$ hematocrit: $\mathrm{MAP}=$ mean arterial pressure $\mathrm{SBP}=$ systolic blood pressure.

${ }^{*}$ Challenges remain for national achievement.

an e-mail Delphi technique allowed our members to create a consensus-derived sepsis care plan based on the CAEP sepsis guidelines. The CAEP sepsis guidelines were approved by the CAEP and met the stringent AGREE instrument for guideline creation.

Sampling of different ED types demonstrates that completion of the level II checklist is attainable only in a tertiary ED, and even then can be challenging. The level I checklist is attainable in almost all community and tertiary EDs. Rural EDs can succeed in completing most of the level I checklist, but there is a strong need for administrative support to be able to obtain early and reliable lactate values and support to obtain technical expertise and adequate materials to reliably place central venous lines. Without completion of 


\begin{tabular}{|c|c|c|c|}
\hline $\begin{array}{l}\text { Given the appropriate time to spend with a septic patient, would you and your ED team } \\
\text { be able to... }\end{array}$ & Rural & Community & Tertiary \\
\hline \multicolumn{4}{|l|}{ Level I checklist } \\
\hline Perform endotracheal intubation & $20 / 20$ & $23 / 23$ & $81 / 81$ \\
\hline Obtain central venous line access (femoral, subclavian, or internal jugular) & $11 / 20$ & $23 / 23$ & $81 / 81$ \\
\hline Perform intravenous fluid administration & $20 / 20$ & $23 / 23$ & $81 / 81$ \\
\hline $\begin{array}{l}\text { Provide vasopressor administration (to achieve MAP }>65 \mathrm{~mm} \mathrm{Hg} \text { and end-organ } \\
\text { perfusion, e.g., dopamine, norepinephrine) }\end{array}$ & $19 / 20$ & $23 / 23$ & $81 / 81$ \\
\hline Obtain serum lactate values & $6 / 20$ & $23 / 23$ & $81 / 81$ \\
\hline $\begin{array}{l}\text { Provide antibiotics within } 60 \text { minutes (based on likely pathogens and patient and local } \\
\text { epidemiology) }\end{array}$ & $19 / 20$ & $23 / 23$ & $81 / 81$ \\
\hline Obtain microbial cultures (e.g., blood, urine, sputum) & $20 / 20$ & $23 / 23$ & $81 / 81$ \\
\hline \multicolumn{4}{|l|}{ Level II checklist } \\
\hline Obtain central venous pressure monitoring (CVP, via upper limb central line) & $2 / 20$ & $10 / 23$ & $65 / 81$ \\
\hline $\begin{array}{l}\text { Obtain central venous gas oxygen saturation value }\left(\mathrm{CVO}_{2} \text { sat, via upper limb central }\right. \\
\text { line) }\end{array}$ & $0 / 20$ & $10 / 23$ & $66 / 81$ \\
\hline Provide fluid resuscitation to achieve a CVP of $8-12 \mathrm{~mm} \mathrm{Hg}$ & $2 / 20$ & $16 / 23$ & $69 / 81$ \\
\hline $\begin{array}{l}\text { Provide inotrope administration to achieve } \mathrm{CVO}_{2} \text { sat }>70 \% \text { and adequate cardiac } \\
\text { output }\end{array}$ & $1 / 20$ & $17 / 23$ & $69 / 81$ \\
\hline Attempt infectious source control (e.g., chest tube, abscess drainage) & $17 / 20$ & $21 / 23$ & $77 / 81$ \\
\hline Provide steroids in patients not responsive to volume/vasopressor & $18 / 20$ & $22 / 23$ & $80 / 81$ \\
\hline Total number of responses & 20 & 23 & 81 \\
\hline
\end{tabular}

these two items, sepsis recognition remains a major obstacle, as does stabilization before transfer. For this reason, lactate values and central lines remain within the level I checklist, but receive an asterisk as a reminder that even these items are not yet nationally achievable. As most Canadian ED physicians practice outside the walls of the tertiary care institution, this creation of a tiered set of checklists gives all ED physicians the ability to appropriately treat their patients in septic shock while addressing the realistic discrepancy that exists among ED environments.

Since the publication of the CAEP sepsis guidelines, other large sepsis studies have been published ${ }^{12-14}$ and many more are under way. ${ }^{7}$ We did not specifically address these newer studies as our intention was to provide a way to apply the CAEP sepsis guidelines in a practical manner. As sepsis care evolves over the next years, both the CAEP sepsis guidelines and checklists will need to be revisited. Readers should note that the sepsis checklists do not rank items based on importance as not even the full range of sepsis literature has been able to achieve this.

Although the process outlined reasonable expectations from the CAEP sepsis guidelines based on ED size, the location of first presentation does not change the ultimate care requirements of the septic patient. As with all other patients, if the centre does not have the resources to provide the level of care required, arrangements must be made for transport to a facility that can provide that level of care.

Competing interests: None declared. 